Pak. j. sci. ind. res. Ser. B: biol. sci. 2019 62B(1) 39-48

\title{
Application of Statistical Quality Control Tools for Monitoring of Pharmaceutical Product Properties
}

\author{
Mostafa Essam Eissa ${ }^{a *}$ and Hassan Shokry Hamed ${ }^{\mathrm{b}}$ \\ ${ }^{a}$ Quality Compliance Department, HP Pharmaceutical Company, Gize, Egypt \\ ${ }^{\mathrm{b}}$ Quality Assurance Department, BP Pharmaceutical Company, East Cairo, Egypt \\ (received March 21, 2017; revised January 20, 2018; accepted February 8, 2018)
}

\begin{abstract}
Pharmaceutical dosage forms are unique products that should meet the highest possible quality standards as they impact the health of the final consumers directly. Safety, identity, strength, purity and quality (SISPQ) are integral properties that should be embedded into the dosage forms through proper implementation of good practices reference guidelines $(\mathrm{G} \times \mathrm{P})$. The current study was aimed to monitor and inspect the quality characteristics of the sustained release capsule product viz: average filling weight, potency determination, dissolution profile, bulk and finished product yield to ensure stability of the pharmaceutical properties of the product and to spot areas of possible improvements provisionally. Statistical process control (SPC) showed that there were intermittent states of out-of-control (OOC) in each monitored properties, although none of the batches showed out-of-specification (OOS) results. Compliance to GxP including good manufacturing practice (GMP) and good laboratory practice (GLP) is important to ensure consistent and predictable performance of the manufacturing process parameter of the product.
\end{abstract}

Keywords: SISPQ, $\mathrm{G} \times \mathrm{P}$, pharmaceutical product, drug properties

\section{Introduction}

Pharmaceutical industry is very critical and delicate industrial field that affects the health of the final consumers through the properties of manufactured products. Regulatory agencies such as United States Food and Drug Administration (USFDA) are working hard to ensure the following of good practices guidelines $(\mathrm{G} \times \mathrm{P})$ within pharmaceutical industry. The goal behind Current Good Manufacturing Practice (cGMP) is to ensure consistency of the manufacturing and processing activities that will deliver the same product with the exact expected properties, in addition to the ability to prove that (Schwalje, 2017). Thus, a reproducible operation (observed through monitoring of statistical quality measurement tools) is capable of delivering products that meet FDA requirements when the good practices standards are conveniently followed.

FDA's 21 Code of Federal Regulation (CFR) part-210 focused on crucial drug properties that should not be violated otherwise the medicinal dosage form will be considered "adulterated" with immediate actions from the regulatory bodies for product, firm and the responsible persons (CFR - 2016a). Five essential characteristics are linked with pharmaceutical product attributes which

*Author for correspondence;

E-mail: mostafaessameissa@yahoo.com are Safety, Identity, Strength, Purity and Quality (SISPQ) (ISPE, 2017).

Complementarily, FDA's 21 CFR part-211 "cGMP for Finished Pharmaceuticals" consists of 11 subparts, each is divided into several subsections that collectively discuss different aspects, conditions and processes that affect and are related to the medicinal products. Broadly speaking, they consist of General Provision, Organization and Personnel, Buildings and Facilities, Equipment, Control of Components and Drug Product Containers and Closures, Production and Process Controls, Packaging and Labeling Control, Holding and Distribution, Laboratory Controls, Records and Reports and finally, Returned and Salvaged Drug Products (CFR, 2016b). Thus, for established processes in the pharmaceutical industry when $\mathrm{G} \times \mathrm{P}$ is followed, the system should be under control and the product quality is reproducible and predictable. However, any deviation from the ordinary process is not part of the normal operation variability which is not acceptable by FDA. This abnormal process variability could be assumed to be spotted by statistical process monitoring.

One of the very common oral pharmaceutical dosage forms is the extended release pellets form (Shah et al., 2015). It offers several advantages such as improving 
patients' compliance by reducing dosing regimen, improve product stability, and maintaining therapeutic concentration (Hayashi et al., 2005). However, the prolonged drug delivery system may suffer from some disadvantages, for instance release rate may be not reproducible between doses for the same product as shown by Hayashi et al. (2005). Thus, thorough monitoring of quality characteristics of this type of products is essential.

Due to such strict regulation, it is obvious that reliance on the compliance to conventional testing criteria of the product only will probably result on the misleading perception for the achievement of $\mathrm{G} \times \mathrm{P}$. On the other hand, the non-conformities may affect adversely the integral system of the pharmaceutical manufacturing before any true and detectable catastrophic excursion may occur. Accordingly, the current work was aimed to investigate the current state of quality of the new pharmaceutical firm, which launched new series of products and elucidate the necessity for improvement with highlights on the defect of the system. This would be accomplished by using statistical process control (SPC) on the gathered data from different processing steps performed on the apparently complying pharmaceutical product.

\section{Materials and Methods}

Quality Assurance (QA) team members from newly established pharmaceutical firm have collected data of manufacturing and analysis of Vitamin $\mathrm{C}$ pellets $500 \mathrm{mg}$ in hard gelatin empty capsule (HGC) size 0 (ECSC, 2017). The new built plant includes large class $\mathrm{D}$ production area for manufacturing solid dosage forms, based in East Cairo Industrial Zone. The clean rooms environment of the manufacturing facility possess continuous monitoring and control system for temperature and humidity through humidity, ventilation and air conditioning (HVAC) units. The inspected results were: dissolution rate (after one (DR1), four (DR4) and eight hours (DR8)), average filling weight, assay of active pharmaceutical ingredient (API), disintegration, bulk yield (BLK) and finished product yield (FN). The tests were claimed to follow pharmacopeial guideline by the quality members of the firm with data obtained from certificates of analysis (CoA) and batch production records (BPR) of the product (USP, 2014). The study is part of larger project that covers the study of several other pharmaceutical products.

The specifications of the product stemmed from National Regulatory Ministry of Health, internal specification which is company-specific and has been harmonized with that provided by the supplier of the raw materials and compendial guideline. Statistical analysis and SPC were done using GraphPad Prism 6 for Windows coupled with XLSTAT Version 2014.5.03 shown in Table 1 and Minitab ${ }^{\circledR}$ 17.1.0, respectively. Application of SPC was done according to Eissa (2016) and Eissa and Abdoh (2016). Statistical analysis was performed as described by Eissa et al. (2015).

The methodology that has been applied in the application of control charts involved the scheme provided by McNeese (2006a). However, modifications have been implemented by the use of the method proposed by Laney in attribute control charts to correct for over- or under-dispersion and avoid problems with data that do not follow Poisson or Binomial distributions (Laney, 2002). Individual-Moving Range (I-MR) chart was used for discrete results that should show certain degree of normality ( $\mathrm{P}>0.05)$ (McNeese, 2009; 2006b) but if not, Laney attribute charts could be implemented.

\section{Results and Discussion}

Detection of out-of-control conditions in pharmaceutical industry using statistical quality control tools has been reported previously (Eissa and Abdoh, 2016). Data trending can show the full state of process and/or inspection characteristic over broader range which may not be observed by depending solely on individual quality control (QC) results. Table 1 shows detailed statistical analysis tabulated as column statistics of the monitored inspection characteristics of 70 manufactured batches of Vitamin C capsules, chronologically arranged. Rate of the inspection properties that pass normality test is $37.5 \%$ and can be processed by I-MR chart type. On the other hand, the remaining inspection properties $(62.5 \%)$ can be processed using Laney U` chart (chart for count-type data) using Minitab, especially that most of them failed to pass either Poisson or Binomial distribution fitting tests (Minitab, 2016). None of the test batches showed out-of-specification (OOS) results and this can be confirmed from Table 1, although some lots of the product demonstrated marginal results. Apart from the possible random variability of the process, deviations may stem from other causes that should be examined, namely: man, operating machines, procedures followed, materials used and testing/analysis system. The investigation should yield corrective action and preventive action (CAPA), if the causative factor has been spotted. 
Data visualization and distribution could be demonstrated in Fig. 1 by using Box Plot (Box-and-Whisker) diagram (Elseviers, 2004). Interestingly, both DR4 and BLK showed outliers date indicated by asterisks "*" at the lower side below the whisker of the lowest value. These points require further analysis and establishment of the relation with those out-of-control points in the following discussed control charts.
Capability histogram shows the probability distribution frequency of the gathered data and it has the advantage of showing the shape of distribution. Moreover, it is tied with process capability analysis if the Upper Specification Limit (USL) and The Lower Specification Limit (LSL) are shown in relation with the position of the process histogram (SYBEQ, 2012). The shape of histogram is very useful tool that aid in the prediction

Table 1. Column statistics generated by statistical software package viz. GraphPad Prism version 6 for Windows and XLSTAT version 2014.5.03 of six inspection quality characteristics and processes

\begin{tabular}{|c|c|c|c|c|c|c|c|c|}
\hline Statistical test & $\begin{array}{l}\text { Average } \\
\text { filling weight }\end{array}$ & Disintegration & Assay & BLK yield & FN yield & DR1 & DR4 & DR8 \\
\hline Minimum & 590 & 4.0 & 96 & 96 & 98 & 21 & 57 & 81 \\
\hline $25 \%$ Percentile & 604 & 6.0 & 99 & 99 & 100 & 25 & 66 & 87 \\
\hline Median & 611 & 8.0 & 100 & 100 & 100 & 28 & 69 & 89 \\
\hline $75 \%$ Percentile & 619 & 9.0 & 101 & 100 & 101 & 31 & 71 & 92 \\
\hline Maximum & 633 & 12 & 103 & 101 & 102 & 35 & 75 & 96 \\
\hline $10 \%$ Percentile & 597 & 5.0 & 98 & 98 & 99 & 23 & 62 & 86 \\
\hline $90 \%$ Percentile & 626 & 9.0 & 102 & 101 & 101 & 33 & 73 & 94 \\
\hline Mean & 611 & 7.4 & 100 & 100 & 100 & 28 & 68 & 89 \\
\hline Std. Deviation & 10 & 1.8 & 1.4 & 0.98 & 0.82 & 3.5 & 4.1 & 3.3 \\
\hline Std. Error of mean & 1.2 & 0.21 & 0.17 & 0.12 & 0.098 & 0.42 & 0.49 & 0.39 \\
\hline Lower $95 \% \mathrm{CI}$ of mean & 609 & 7.0 & 99 & 99 & 100 & 27 & 67 & 89 \\
\hline Upper $95 \% \mathrm{CI}$ of mean & 614 & 7.8 & 100 & 100 & 100 & 29 & 69 & 90 \\
\hline Lower $95 \% \mathrm{CI}$ of median & 608 & 7.0 & 99 & 100 & 100 & 27 & 67 & 88 \\
\hline \multirow[t]{2}{*}{ Upper $95 \% \mathrm{CI}$ of median } & 614 & 8.0 & 100 & 100 & 100 & 29 & 70 & 91 \\
\hline & \multicolumn{8}{|c|}{ Shapiro-Wilk normality test } \\
\hline W & 0.98 & 0.94 & 0.99 & 0.91 & 0.99 & 0.96 & 0.95 & 0.95 \\
\hline$P$ value & 0.5186 & 0.0024 & 0.8157 & $<0.0001$ & 0.9563 & 0.0298 & 0.0051 & 0.0098 \\
\hline Passed normality test $(\alpha=0.05)$ ? & Yes & No & Yes & No & Yes & No & No & No \\
\hline \multirow[t]{2}{*}{$P$ value summary } & ns & $* *$ & ns & $* * * *$ & ns & * & $* *$ & $* *$ \\
\hline & \multicolumn{8}{|c|}{ Moments estimation method at $5 \%$ significance level } \\
\hline \multirow[t]{2}{*}{ Poisson } & nd & + & - & - & + & - & - & - \\
\hline & \multicolumn{8}{|c|}{ Moments estimation method at $5 \%$ significance level } \\
\hline \multirow[t]{2}{*}{ Binomial } & nd & - & - & - & - & - & - & - \\
\hline & \multicolumn{8}{|c|}{ Maximum probability estimation method for automatic best fitting distribution at $5 \%$ significance level } \\
\hline Closest fitting distribution & Log-normal & Weibull II & $\begin{array}{l}\text { Generalized } \\
\text { extreme value } \\
(\mathrm{GEV})\end{array}$ & Logistic $^{\Psi}$ & Logistic & nd & nd & $\begin{array}{l}\text { Log- } \\
\text { normal }\end{array}$ \\
\hline Coefficient of variation & $1.71 \%$ & $23.92 \%$ & $1.44 \%$ & $0.98 \%$ & $0.82 \%$ & $12.58 \%$ & $6.07 \%$ & $3.68 \%$ \\
\hline Geometric mean & 611 & 7.2 & 100 & 100 & 100 & 28 & 68 & 89 \\
\hline Lower $95 \% \mathrm{CI}$ of geo. mean & 609 & 6.8 & 99 & 99 & 100 & 27 & 67 & 89 \\
\hline Upper $95 \%$ CI of geo. mean & 614 & 7.6 & 100 & 100 & 100 & 29 & 69 & 90 \\
\hline Skewness & 0.0 & -0.031 & 0.0 & -1.1 & 0.0 & 0.051 & -0.75 & 0.030 \\
\hline Kurtosis & -0.68 & -0.55 & -0.21 & 1.4 & -0.0094 & -1.0 & 0.32 & -0.18 \\
\hline Sum & 42789 & 519 & 6976 & 6971 & 7009 & 1965 & 4770 & 6260 \\
\hline
\end{tabular}

ns = not significant; $\mathrm{nd}=$ not determined; $+=$ fit; - = not fit; $\Psi=$ Did not fit the distribution by Chi-Square test; $\mathrm{CI}=$ confidence interval. 
of the process behavior (Tague, 2005). For instance, erratic bins in Fig. 2b \& D i.e., DR1 and DR8 are indication of processes that have been executed abnormally different from the normal ones. In contrast to average filling weight, assay and $\mathrm{FN}$, which approximated the bell-shaped distribution (Fig. 2F, 3 and 4), the other distributions showed significant distortion to the normal distribution due to unusual causes that require further investigation. For example, Fig. 2A, C and E showed pattern that is close to two interfering distributions, one major and the other is minor, which may indicate two distinct processes performed for one operation. Because of their shape, they are called "Double-Peaked or Bimodal Distribution". Moreover, Fig. $2 \mathrm{C}$ is partially truncated from the right side i.e.
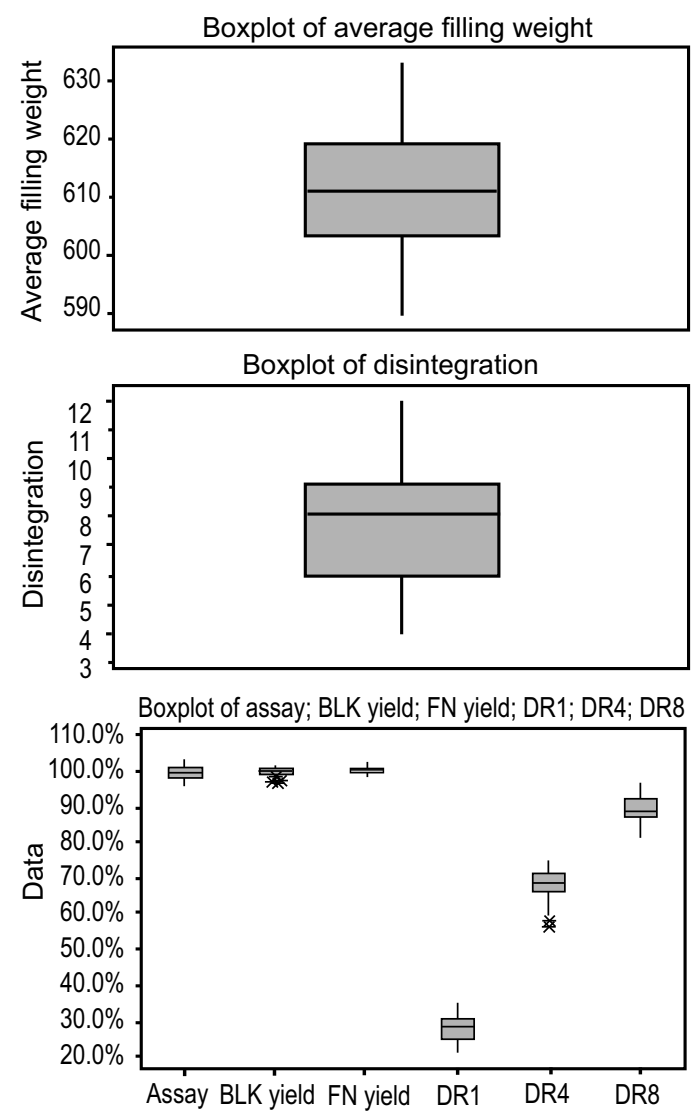

Fig. 1. Box-and-Whisker diagram of average filling weight, disintegration, assay of active pharmaceutical ingredient (API), bulk (BLK) yield, finished (FN) yield, dissolution rate after one hour (DR1), after four hours (DR4) and after eight hours (DR8). [generated using Minitab ${ }^{\circledR}$ 17.1.0]. toward the USL and this action is usually due to external factors that are not related to the normal process such as selective screening, preferential inspection and/or a review operation. Figure $2 \mathrm{~B}$ and $\mathrm{D}$ are close to what is called "Multimodal Distribution" (ASQ, 2017). However, they did not show typical plateau appearance, which may be because of processes that appeared to predominate the others in single inspected property. Most importantly, the processes that did not show distribution close to Gaussian one, demonstrated non-consistent activities in the manufactured product cycle that requires immediate corrective actions and to implement strict standard operations.

The process capability six pack diagram of the average filling weight is demonstrated in Fig. 3. It was noted that the general trend line is decreasing with progression with production batches - which may be attributed to machine wearing or maintenance with scattered out-ofcontrol lots due to early warning of potential process shift followed by true drift in the average. This could be viewed in the capability histogram, where the process is shifted toward the left side i.e., LSL. Severe fluctuation in process variation between Batch 46 and 48 is abnormal and attributable to extraneous factors rather than inherited operational variability. Similar observations could be concluded in Fig. 4 for the assay of API except that the general trend line is increasing and the out-of-control batches require planned laboratory investigation.

Assessment of process capability visually could be determined from Fig. 3-5. In general, long term process spread is greater than the short term one. However, it is the magnitude of that difference that determines the process stability over prolonged period (Scibilia, 2015). Interestingly, Fig. 4 demonstrated that the assay results have met the requirements; nevertheless there were ten batches out-of-control. In the same line, Fig. 3 showed that process spread still confined within the specifications (specs) spread but the overall operation is shifted towards the lower limit which signal warning alarm concerning the future possible deviation in the average filling weight. Figure 5B of DR4 showed strong shift above the USL indicating serious drift of the process that could be exaggerated overtime. Fig. 5A and C for D8 and DR1, respectively are approximately centralized but did not meet the specifications over long run. Generally, the dissolution test did not show satisfactory performance and require corrective action after elucidating the root cause either the manufacturer of the Vitamin $C$ pellets and/or laboratory issue. 



Fig. 2(A-F). Capability histogram with upper specification limit (USL) and lower specification limit (LSL) for: disintegration test (A), DR8 (B), DR4 (C), DR1 (D), BLK (E) and FN (F). [generated using Minitab $^{\circledR}$ 17.1.0].

Control charts in Fig. 6-11 demonstrated the remaining inspection processes with similar out-of-control points as in Fig. 3 and 4 but for different batches and hence very low correlation (if any) was found between these parameters except normally for dissolution points.
Surprisingly, while disintegration of capsules was within the specification, yet the number of deviating points was remarkably high if compared with other control charts in this study (about 27 batches showed shift in the disintegration mean value) which requires further 


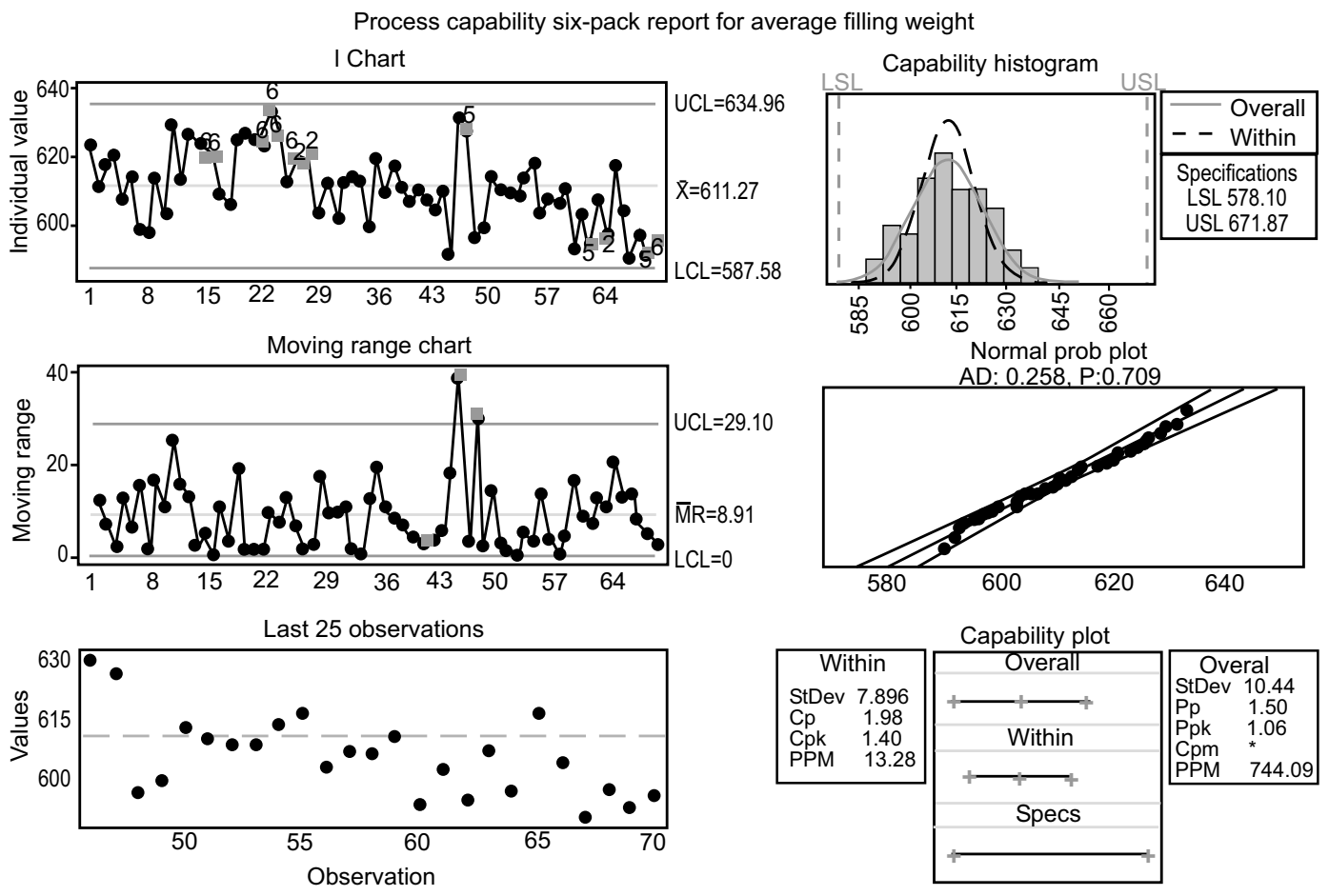

Fig. 3. Statistical quality tools applied on normally distributed average filling weight data of ascorbic acid pellets. [generated using Minitab ${ }^{\circledR}$ 17.1.0].

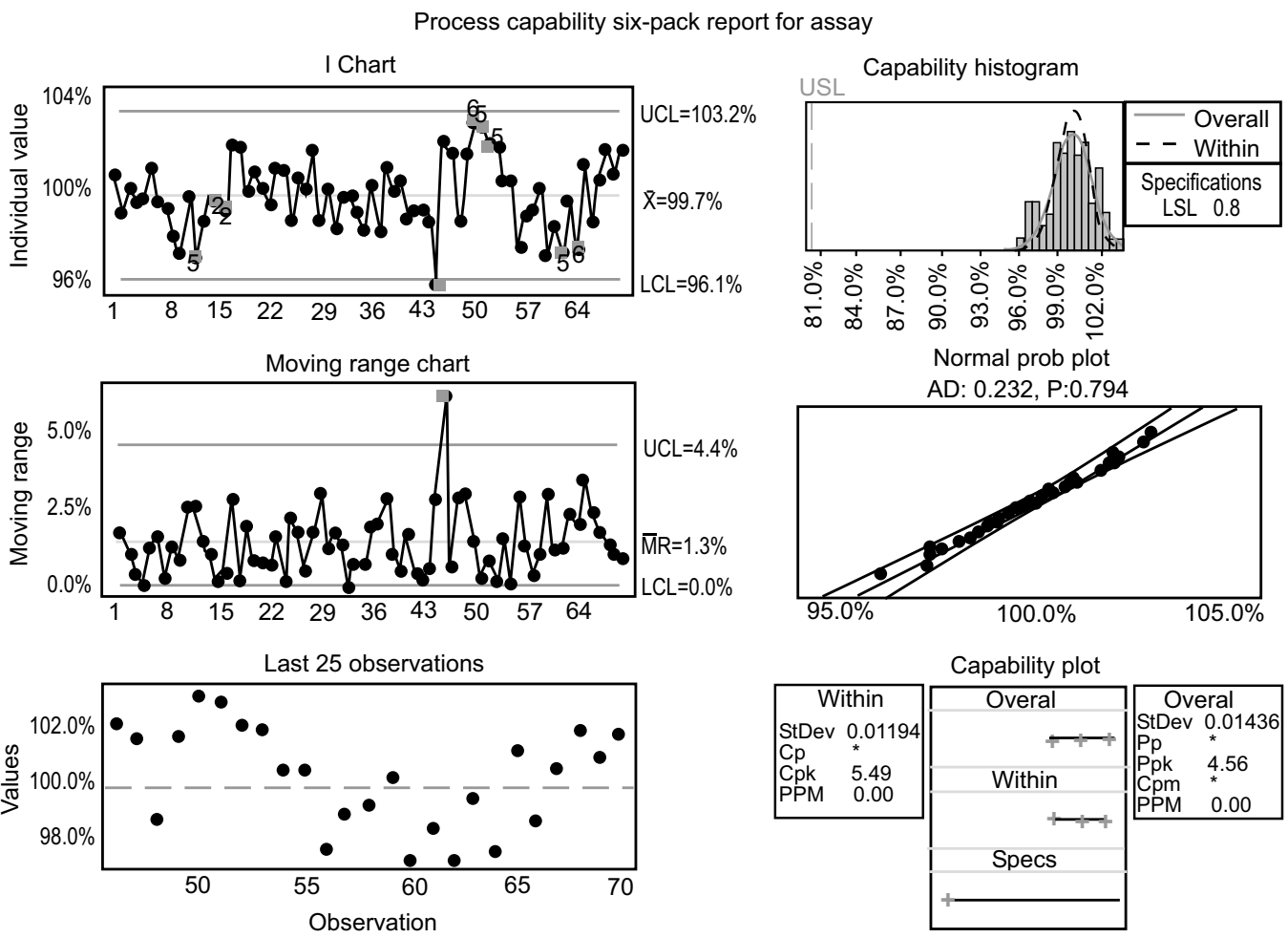

Fig. 4. Statistical quality tools applied on normally distributed data for assay of ascorbic acid capsule. [generated using Minitab ${ }^{\circledR}$ 17.1.0]. 

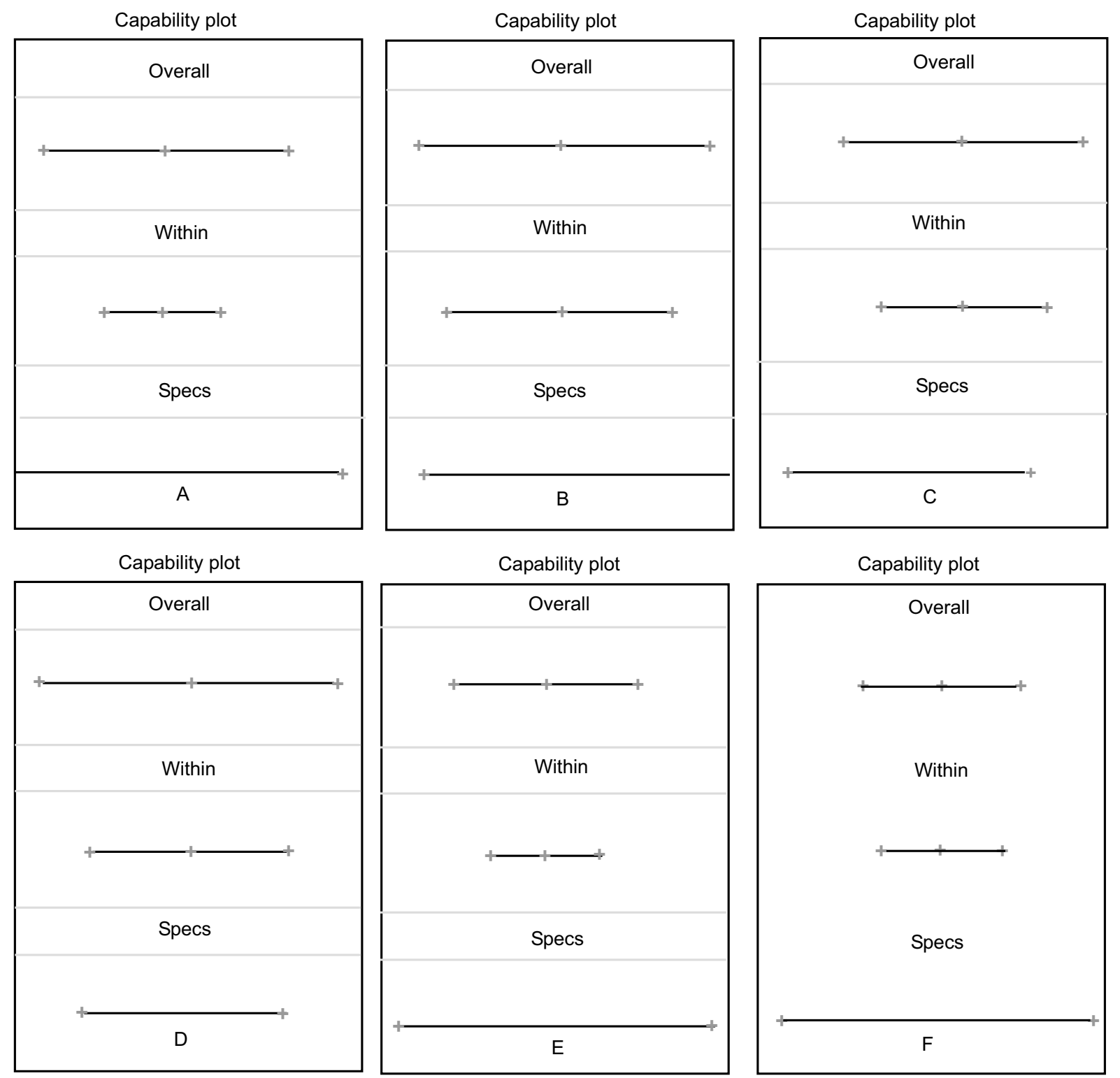

Fig. 5(A-F). Capability plot showing short (within) and long (overall) process stability in addition to the shift in the inspected parameter in relation to the internal specifications (specs) of the product. Inspection characteristics are: disintegration (A), DR8 (B), DR4 (C), DR1 (D), BLK (E) and FN (F). [generated using Minitab ${ }^{\circledR}$ 17.1.0].

investigation with the possibility of the impact of changing the suppliers alternatively could impact this property. Spearman correlation was conducted using GraphPad Prism version 6. With exception of FN, all other processes showed decreasing trend line. It should be noted that capability histogram and plot of FN were shown separately from Fig. 8 in Fig. 2F and Fig. 5F for comparison with that of BLK and to show the degree of difference in efficiency of these two related processes.
It could be concluded that the product manufacturing cycle need improvements, although all tested batches were acceptable and met the specifications. SPC provided useful tool to detect conformance of the processes and compliance to $\mathrm{G} \times \mathrm{P}$ rules before any true excursion would be evident through product. The provided study demonstrated that the quality implementation and attitude is crucial through the system. Accordingly, it will be translated into the product which is the final outcome 


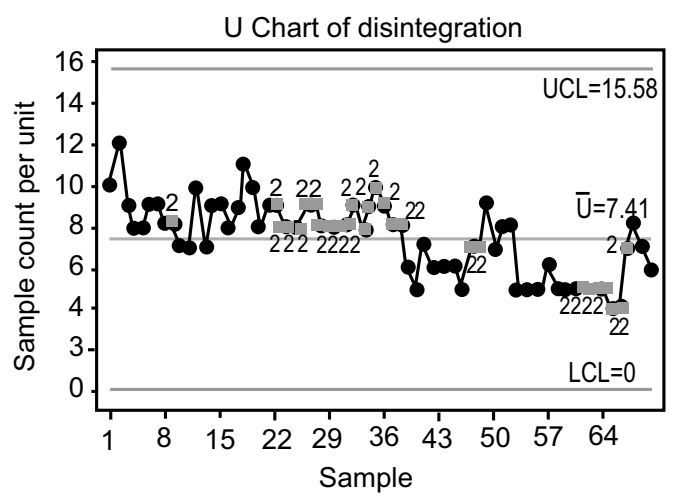

Fig. 6. Laney-modified attribute control chart of disintegration.

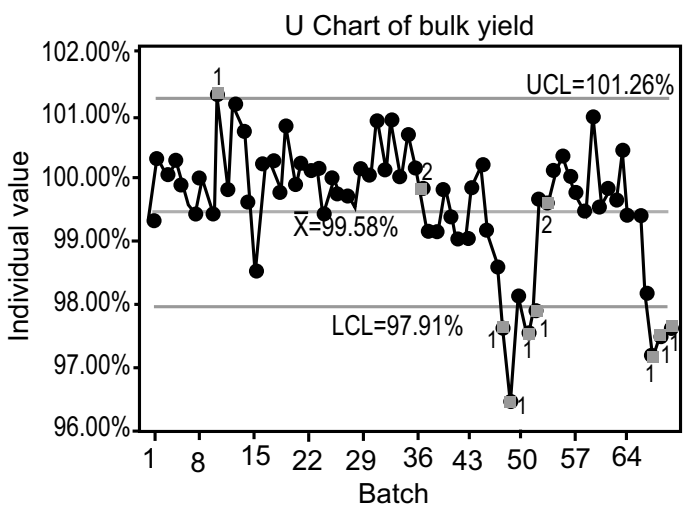

Fig. 7. Laney-modified attribute control chart of BLK.

Process capability six-pack report for FN yield
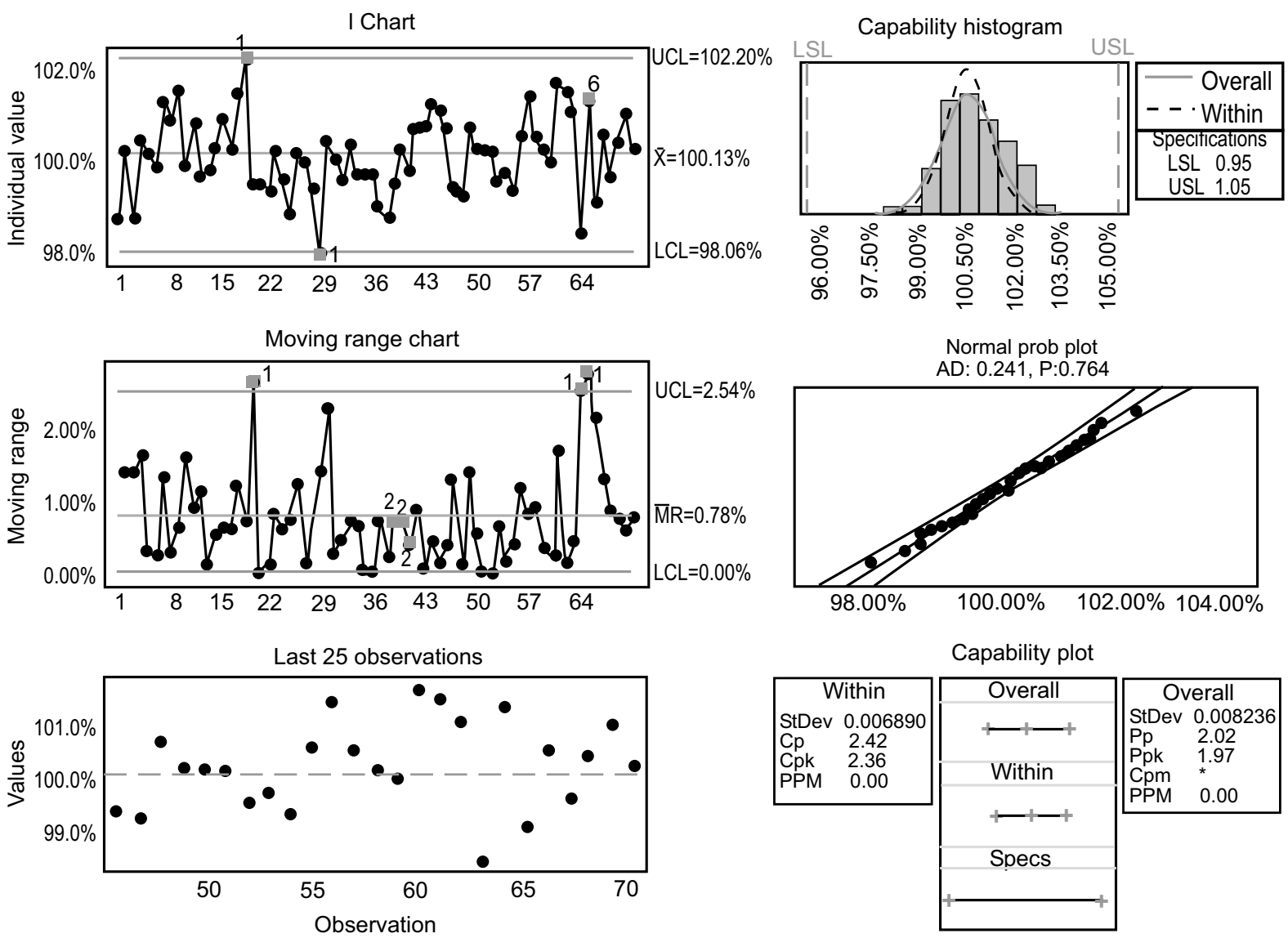

Fig. 8. Statistical quality tools applied on normally distributed data for FN. [generated using Minitab ${ }^{\circledR}$ 17.1.0].

of this rigorous and strict control. The reliance on product only to judge quality is misleading and simply may hide a continuously deviating system that could lead finally to catastrophic outcome with severe financial and reputation loss in competing world of the pharmaceutical industry. 


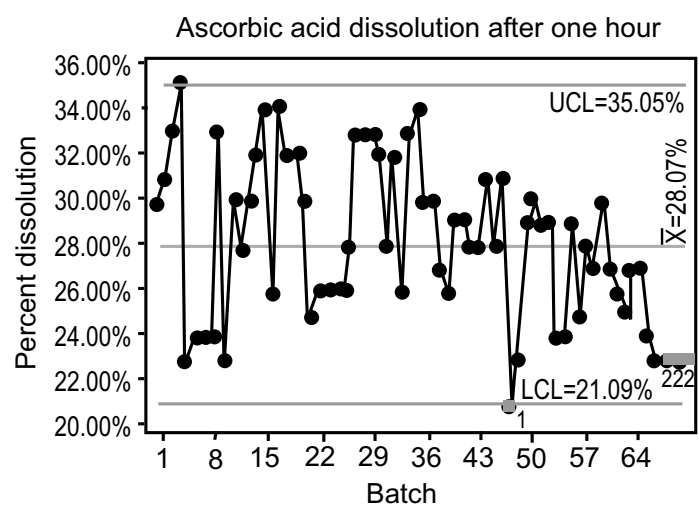

Fig. 9. Laney-modified attribute control chart of DR1.

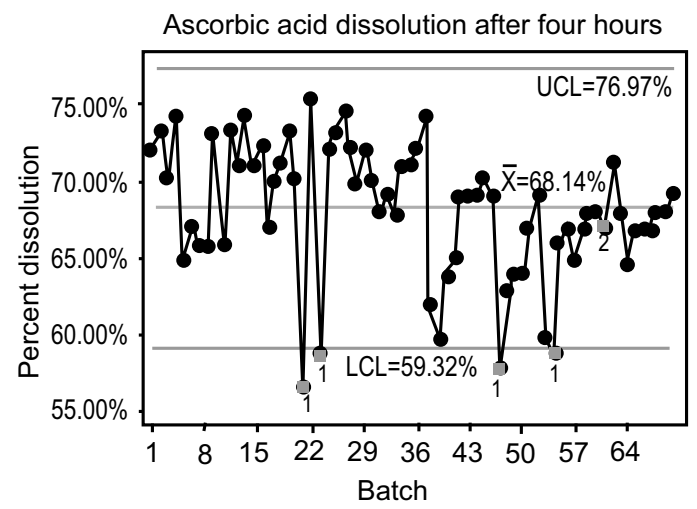

Fig. 10. Laney-modified attribute control chart of DR4.

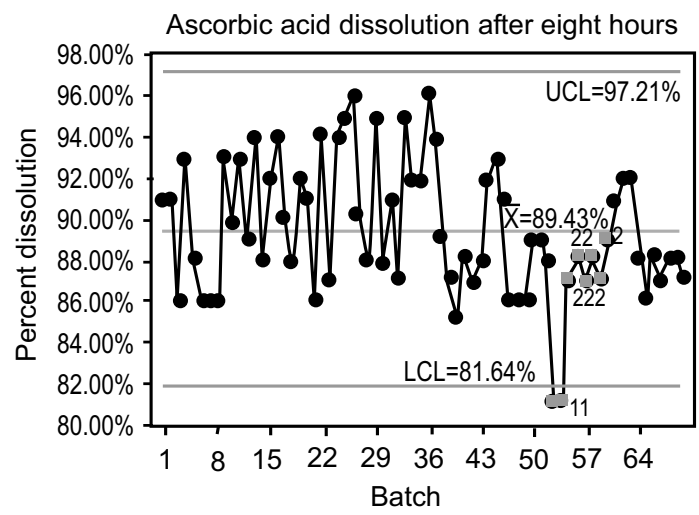

Fig. 11. Laney-modified attribute control chart of DR8.

Conflict of Interest. The authors declare no conflict of interest.

\section{References}

ASQ, 2017. Asq.org. Retrieved 15 March 2017, from http://asq.org/learn-about-quality/data-collectionanalysis-tools/overview/histogram $2 . \mathrm{html}$

CFR, 2016a. Current good manufacturing practice in manufacturing, processing, packing, or holding of drugs, General, Code of Federal Regulations Title 21/Part 210. Accessdata.fda.gov. Retrieved 11 March 2017, from http://www.accessdata.fda. gov/scripts/cdrh/cfdocs/cfcfr/CFRSearch.cfm?C FRPart $=210 \&$ showFR $=1$

CFR, 2016b. Current good manufacturing practice for finished pharmaceuticals, Code of Federal Regulations Title 21/Part 211. Accessdata.fda.gov. Retrieved 11 March 2017, from http://www. accessdata.fda.gov/scripts/cdrh/cfdocs/cfcfr/CFR Search.cfm?CFRPart=210\&showFR $=1$

ECSC, 2017. Empty Capsule Size Chart, Capsuline.com. Retrieved 11 March 2017, from https://www. capsuline.com/empty-capsule-size-chart/

Eissa, M. 2016. Shewhart control chart in microbiological quality control of purified water and its use in quantitative risk evaluation. UK Journal Of Pharmaceutical Biosciences, 4: 45-51.

Eissa, M., Abdoh, A. 2016. Evaluation of quality characteristics and process stability for pharmaceutical dosage form using attribute control charts. International Journal of Advances in Medical Sciences, 1: 9-15.

Eissa, M., Seif, M., Fares, M. 2015. Assessment of purified water quality in pharmaceutical facility using six sigma tools. International Journal of Quality Assurance, 6: 54-72.

Elseviers, M.M. 2004. Statistics corner: The box plot: An alternative way to present a distribution of observations. European Dialysis and Transplant Nursing Association/European Renal Care Association, 30: 114-116. http://dx.doi.org/10.1111/j.17556686.2004.tb00345.x

Hayashi, T., Kanbe, H., Okada, M., Suzuki, M., Ikeda, Y., Onuki, Y., Kaneko, T., Sonobe, T. 2005. Formulation study and drug release mechanism of a new theophylline sustained-release preparation. International Journal of Pharmaceutics, 304: 91-101.

ISPE, 2017. International Society for Pharmaceutical Engineering (ISPE) Glossary of Pharmaceutical and Biotechnology Terminology. Ispe.org. Retrieved 11 March 2017, from http://www.ispe.org/glossary? term=SISPQ 
Laney, D. 2002. Improved control charts for attributes. Quality Engineering, 14: 531-537. http://dx.doi.org/ 10.1081/qen-120003555

McNeese, B. 2009. Attribute Control Charts Overview BPI Consulting. Spcforexcel.com. Retrieved 14 March 2017, from https://www.spcforexcel.com/ knowledge/attribute-control-charts/attribute-controlcharts-overview

McNeese, B. 2006a. Selecting the Right Control Chart BPI Consulting. Spcforexcel.com. Retrieved 14 March 2017, from https://www.spcforexcel.com/ knowledge/control-chart-basics/selecting-rightcontrol-chart

McNeese, B. 2006b. Individuals Control Charts $\mid$ BPI Consulting. Spcforexcel.com. Retrieved 14 March 2017, from https://www.spcforexcel.com/ knowledge/variable-control-charts/individualscontrol-charts

Minitab. 2016. Support.minitab.com. Retrieved 14 March 2017, from http://support.minitab.com/enus/minitab/17/topic-library/quality-tools/controlcharts/understanding-attributes-control-charts/whatis-a-laney-u-chart/

Schwalje, H. 2017. Good Manufacturing Practice
Background and Overview-Emerson Process Experts, Emerson Process Experts. Retrieved 10 March 2017, from https://www.emersonprocess xperts.com/2013/08/good-manufacturing-practicebackground-and-overview/

Scibilia, B. 2015. A Simple Guide to Between/Within Capability Minitab. Blog.minitab.com. Retrieved 15 March 2017, from http://blog.minitab.com/blog/ applying-statistics-in-quality-projects/a-simpleguide-to-between-within-capability

Shah, S., Shah, P.B., Patel M.S., Patel, M.R. 2015. A review on extended release drug delivery system and multiparticulate system. World Journal of Pharmaceutical Research, 4990: 724-747.

SYBEQ, 2012. Histogram \& Capability Analysis. System for Better Quality, Sybeq.blogspot. com.eg. Retrieved 14 March 2017, from http://sybeq. blogspot.com.eg/2012/05/histogram-capabilityanalysis.html

Tague, N. 2005. The Quality Toolbox, $1^{\text {st }}$ edition, pp. 292-299, ASQ Quality Press, Milwaukee, USA. USP, 2014. US Pharmacopoeia National Formulary, USP 37 NF32. Columbia University Press, New York, USA. 